\title{
Prevalence of Non albicans Candida Vaginal Infection among Women Attending the Obstetrics and Gynaecology Department of a Tertiary care Hospital
}

\author{
V. Sangamithra*, Susan, Radha Madhavan and Gomathi
}

\author{
Department of Microbiology, SRM Medical College \& RI, Chennai, India \\ *Corresponding author
}

\begin{tabular}{|c|c|}
\hline & A B S T R A C T \\
\hline Keywords & \multirow{7}{*}{$\begin{array}{l}\text { Candida species are component of normal flora of human being. They are commonly } \\
\text { found on the skin throughout gastrointestinal tract and female genital tract particularly } \\
\text { higher in vagina during pregnancy. Vaginal yeast infection, also known as vaginal } \\
\text { candidiasis, genital candidiasis, or vulvovaginal candidiasis (VVC), is an infection } \\
\text { affecting women of all age groups. The increase in the incidence of Candida species } \\
\text { over the past two decades is significant and non-albicans Candida species continue to } \\
\text { replace C. albicans at most of the clinical sites. Diabetes, immunosuppressive disease } \\
\text { or therapies and neutropenia are common risk factors. Other risk factors include the } \\
\text { use of oral contraceptives, corticosteroids etc. (Chow et al., 2008). Candida species } \\
\text { can cause a wide spectrum of clinical disease involving mucous membrane mainly, } \\
\text { skin and nails. The present study was undertaken to assess the prevalence of vaginal } \\
\text { infections caused by non albicans Candida and to look for the antifungal susceptibility } \\
\text { pattern of the isolates. }\end{array}$} \\
\hline Candida albicans, & \\
\hline Vulvovaginal & \\
\hline Candidiasis, & \\
\hline CHROM Agar. & \\
\hline Article Info & \\
\hline $\begin{array}{l}\text { Accepted: } \\
\text { 12 April } 2017 \\
\text { Available Online: } \\
10 \text { May } 2017\end{array}$ & \\
\hline
\end{tabular}

\section{Introduction}

Vulvovaginal Candidiasis (VVC) represents a spectrum of disease. Although it is the second most common vaginal infection, $\mathrm{VVC}$ is a non notifiable disease and has been excluded from the ranks of sexually transmitted diseases. For many years patients with vaginal Candida were categorized into two groups: Asymptomatic carriers of Candida (colonization) and Symptomatic disease (Vaginal Candidiasis). More recently, the concept of "VVC" has replaced these distinct categories and the "vulvar", often dominant component of symptomatic infection.
It is classified as uncomplicated (Sporadic or infrequent VVC, Mild to moderate VVC, Likely to be caused by Candida albicans and in normal, non pregnant women), complicated [recurrent VVC, severe VVC commonly caused by Non albicans Candidiasis occurring in abnormal host (e.g., uncontrollable diabetes, debilitation, or immunosuppression)].

Seventy-five percent of all women develop a yeast infection at some point during their lives. The infection may be acute or chronic, superficial or deep and its clinical spectrum is 
wide. It is found mainly as secondary infection in individuals with some underlying immunocompromised conditions and very rarely as the primary disease. The fungus most commonly associated with vaginal yeast infection is called Candida albicans which are normally found in warm and moist areas of the body. Cases of sporadic and recurrent VVC caused by nonalbicans species of Candida are also on the rise. The possible reason for the apparent increase in non albicans Candida Vulvovaginitis may be the increased use of antimycotics, which may be used inappropriately and frequently as a short, incomplete course of therapy, eliminating the more sensitive Candida albicans and selecting for more azole resistant non albicans Candida species.

The vaginal $\mathrm{pH}$ is typically 4 to 4.5 (normal), which distinguishes candidiasis from trichomoniasis or bacterial vaginosis (where the $\mathrm{pH}$ is elevated), there is a thick, white, cottage cheese-like discharge associated with pruritus in cases of vulvovaginal candidiasis. Risk factors associated with vaginal candidiasis include age, race, elevated estrogen, diabetes mellitus, contraception, use of antibiotics and immune suppression. Recurring yeast infections may be a sign of a serious disease such diabetes, leukemia or AIDS.

Over 200 species of Candida exist in nature; so far, only a few species have been associated with the disease in humans. The medically significant Candida species includes -Candida albicans (the most common species identified), Candida glabrata (previously known as Torulopsis glabrata), Candida parapsilosis, Candida tropicalis, Candida krusei, Candida kefyr (previously known as Candida pseudotropicalis), Candida guilliermondii, Candida lusitaniae, Candida dubliniensis, Candida rugosa, Candida viswanathii. Among these species of genus Candida,
Candida albicans accounts for most cases of systemic candidiasis but infections due to $C$. krusei, C. glabrata, C. tropicalis, C. kefyr, $C$. guilliermondii, C. parapsilosis, C. lusitania and $C$. rugosa are increasingly being reported (Textbook of Medical Mycology, Jagdish Chander, $3^{\text {rd }}$ edition). In spite of the basic morphological similarity between various species, a number of characteristics, which are species specific, are evident on Corn Meal Agar (CMA). Sugar fermentation and assimilation are the main methods for speciation of Candida.

Candida species are responsible for most of the frequently encountered opportunistic fungal infection. The increase in the incidence of Candida species over the past two decades is significant and non-albicans Candida species continue to replace C.albicans at most of the clinical sites like blood stream infections. Although C. albicans remains the most common causative agent of both superficial and deep fungal infection, an increase in the Candida non-albicans species like C. tropicalis, C. krusei, C. glabrata and $C$. parapsilosis have been documented (Eggimann et al., 2003). The ideal antifungal agents need to be effective against a broad spectrum of organisms, including all the medically important yeast of the genus Candida.

The most important classes of antifungal agents effective against Candida species are the azole and the polyene antifungal agents. Fluconazole is the most commonly used azole fungal agents and has been proven efficacious in the treatment of both superficial and systemic fungal disease.

Candida krusei, Candida glabrata, Candida dubliniensis, Candida tropicalisare resistant to Fluconazole. Candida albicans, Candida glabrata and Candida krusei are resistant to Amphotericin B. 
The main aim of this study to isolate and identify Candida from high vaginal swabs, to identify various Candida species, to assess the prevalence of non albicans Candida Vaginal infection among symptomatic pregnant and non-pregnant women coming to the Obstetrics and Gynaecology department of SRM hospital. To determine the commonest non albicans Candida species causing Vaginal Candidiasis to find out the prevalence of vaginal candidiasis among various age groups. Also to find out the antifungal susceptibility test of the isolates

\section{Materials and Methods}

A prospective study was carried out from May 2010 to February 2011 to study the prevalence of Non albicans Candida Vaginal infection among symptomatic patients coming to the OG department of SRM hospital. A detailed history which included patients name, age, sex, date of admission, ward, a brief clinical history, the frequency of menstrual cycle, underlying diseases, antibiotic therapy, presence of any risk factors, steroid intake, pregnancy, diadetes mellitus, use of contraceptives and other symptoms suggestive of vaginal candidiasis like itching, colour and odour of vaginal discharge etc were collected from the patients. High vaginal swabs were collected aseptically from the patients by standard techniques.

\section{Specimen processing}

A direct smear for assessing of the cellularity and presence of organism was carried out in all cases. The media for the study were purchased from Himedia (Mumbai).The media and the biochemicals were prepared following standard procedures (Textbook of Medical Microbiology, Jagdish Chander, $3^{\text {rd }}$ edition) (Annexure 2). Each batch of media and biochemicals were tested with suitable controls and was utilized only if it was satisfactory.
The primary isolation of the specimen was done on Sabouraud Dextrose Agar (SDA) with and without antibiotics (gentamicin) and incubated overnight at $37^{\circ} \mathrm{C}$. The isolates produced cream coloured and smooth colonies on SDA. The isolates were subjected to gram stain which showed gram positive budding yeast cells. A preliminary identification of the isolates was done by performing the germ tube test and inoculating on Corn Meal Agar (CMA) which differentiated Candida albicans from Candida non albicans.

\section{Biochemical reactions}

Once presumptively identified as belonging to the genus Candida, the organism was subjected to further identification upto species level based on characteristic biochemical tests which includes Sugar fermentation test, Sugar assimilation test, Pellicle formation in Sabouraud Dextrose Broth (SDB), Urease test and CHROM Agar.

\section{Antifungal susceptibility testing}

Antifungal susceptibility testing was performed by NCCLS M44-A Disc diffusion method.18 Inoculum was prepared by picking five distinct colonies of approximately $1 \mathrm{~mm}$ in diameter from a $24 \mathrm{~h}$ old culture of Candida species. Colonies were suspended in $5 \mathrm{ml}$ of sterile saline and its turbidity was adjusted visually with the transmittance to that produced by a $0.5 \mathrm{McFarland}$ standard. Inoculation of test plates were done with a sterile cotton swab dipped into the suspension. The dried surface of a sterile Mueller-Hinton + GMB (glucose and methylene blue) agar plate was inoculated by evenly streaking the swab over the entire agar surface. Anti fungal disks of Fluconazole, Itraconazole and Amphotericin B were dispensed onto the surface of the inoculated agar plate. The plates were inverted and 
placed in an incubator set to $35^{\circ} \mathrm{C}$ within 15 minutes after the anti fungal disks were applied. The inoculated plates were examined after 20 to 24 hours of incubation. The zone of inhibition was measured and the result is recorded as susceptible. The susceptible category implies that an infection due to the strain may be appropriately treated with the dose of antimicrobial agent recommended for that type of infection and infecting species.

Susceptible-dose dependent (S-DD): The susceptible-dose dependent category includes isolates with antimicrobial agent MICs that approach usually attainable blood and tissue levels and for which response rates may be lower than for susceptible isolates.

Resistant (R): Resistant strains are those that are not inhibited by the usually achievable concentrations of the agent with normal dosage schedules.

\section{Results and Discussion}

The present study was carried out from May 2010 to February 2011 in the Obstetrics and Gynaecology department of SRM Medical College Hospital which is a tertiary care centre. A total of 68 samples were collected from symptomatic patients attending the outpatient department of Obstetrics and Gynaecology.

The demographic profile of the study subjects showed (Figure 1) symptomatic patients were common in the reproductive age group and least among the adolescent and premenopausal age group. Of the total 68 samples collected, majority of the patients $(n=51)$ were between 21 to 40 years of age.

The distribution of Candida species (Figure 2) showed a majority of Candida non albicans which was $71 \%(\mathrm{n}=17)$ and Candida albicans constitute only $29 \%(\mathrm{n}=7)$
The distribution of non albicans Candida among pregnant and non pregnant women (Table 1). Among the pregnant women $(n=25)$ Candida albicans were isolated from 4 samples and Candida non albicans from 6 samples. Likewise in non pregnant women $(\mathrm{n}=43)$ only 3 samples grew Candida albicans and Candida non albicans was isolated from 11 samples.

Figure 3 depicts the distribution of species among Candida non albicans. Among the Candidanon albicans $(\mathrm{n}=17), \quad$ Candida parapsilosis was the commonest isolate $41 \%$ $(\mathrm{n}=7)$, followed by Candida krusei $23 \%$ $(\mathrm{n}=4)$, Candida glabrata $18 \%(\mathrm{n}=3)$, Candida kefyr $12 \%(\mathrm{n}=2)$ and Candida tropicalis $6 \%$ $(\mathrm{n}=1)$.

Figure 4 depicts the percentage of diabetic and non diabetic patients. Among the total of 68 symptomatic patients who attended the obstetrics and gynaecology department of SRM hospital $1.5 \% \quad(n=1)$ was diabetic whereas $98.5 \% \quad(n=67)$ were non diabetic patients.

Figure 5 depicts the percentage of antibiotic user and nonuser. Among the total of 68 symptomatic patients who attended the obstetrics and gynaecology department of SRM hospital 6\% $(n=4)$ were antibiotic users whereas $94 \% \quad(n=64)$ were antibiotic nonusers.

Table 2 shows the distribution of non albicans Candida isolates based on sugar fermentation. In the present study, out of the 24 positive samples, glucose was found to be fermented by all the species of Candida, maltose was fermented by Candida albicans, Candida kefyr and Candida tropicalis and sucrose was fermented by both Candida kefyr and Candida tropicalis.

Table 3 shows the distribution based on sugar assimilation. Candida parapsilosis $(n=7)$ did 
not assimilate lactose and dulcitol, on the other hand Glucose and xylose were assimilated by Candida krusei $(\mathrm{n}=4)$ and Candida glabrata $(\mathrm{n}=3)$ assimilated glucose, xylose and dulcitol. Candida kefyr $(\mathrm{n}=2)$ did not assimilate sucrose and dulcitol; likewise dulcitol and lactose were not assimilated by Candida tropicalis $(\mathrm{n}=1)$.

\section{Growth on CHROM agar}

Among the 24 isolates grown on CHROM agar Candida albicans $(\mathrm{n}=7)$ produced light green coloured colonies, whereas Candida parapsilosis $(\mathrm{n}=7)$ showed cream to pale pink coloured colonies. Likewise Candida krusei $(\mathrm{n}=4)$ and Candida glabrata $(\mathrm{n}=3)$ produced pink and pink to purple coloured colonies. Candida kefyr $(\mathrm{n}=2)$ and Candida tropicalis $(\mathrm{n}=1)$ produced pink or purple coloured and dark blue coloured colonies.

Table 4 shows susceptibility pattern of Candida speices to Fluconazole, Itraconazole and Amphotericine B. Out of all species of Candida NON ALBICANS, resistance to Fluconazole, Itraconazole and Amphotericine $\mathrm{B}$ in 5, 5 and 1 case respectively. Resistance to Fluconazole and Itriconazole were found maximum in Candida krusei followed by Candida parapsilosis.

Table.1 Prevalent of $C$. albicans and Non albicans Candida

\begin{tabular}{|l|l|l|}
\hline & Pregnant & Non pregnant \\
\hline Non albicans Candida & 6 & 11 \\
\hline Candida albicans & 4 & 3 \\
\hline
\end{tabular}

Table.2 Distribution of isolates based on sugar fermentation

\begin{tabular}{|l|l|l|l|l|l|}
\hline Isolate & Total & Glucose & Maltose & Sucrose & Lactose \\
\hline C. parapsilosis & $\mathbf{7}$ & $\mathbf{7}$ & - & - & - \\
\hline C. krusei & $\mathbf{4}$ & $\mathbf{4}$ & - & - & - \\
\hline C. glabrata & $\mathbf{3}$ & $\mathbf{3}$ & - & - & - \\
\hline C. kefyr & $\mathbf{2}$ & $\mathbf{2}$ & $\mathbf{2}$ & $\mathbf{2}$ & - \\
\hline C. tropicalis & $\mathbf{1}$ & $\mathbf{1}$ & $\mathbf{1}$ & $\mathbf{1}$ & - \\
\hline
\end{tabular}

Table.3 Distribution of non albicans candida isolates based on sugar assimilation

\begin{tabular}{|l|l|l|l|l|l|l|l|}
\hline Isolate & Total & Glu & Mal & Suc & Lac & Xyl & Dul \\
\hline C. parapsilosis & $\mathbf{7}$ & + & + & + & - & + & - \\
\hline C. krusei & 4 & + & - & - & - & + & - \\
\hline C. glabrata & 3 & + & - & - & - & + & + \\
\hline C. kefyr & 2 & + & - & + & + & + & - \\
\hline C. tropicalis & 1 & + & + & + & - & + & - \\
\hline
\end{tabular}


Table.4 Susceptibility pattern of nonalbicans Candida

\begin{tabular}{|l|l|l|l|l|l|l|l|}
\hline Isolates & $\begin{array}{l}\text { No: of } \\
\text { isolates }\end{array}$ & \multicolumn{2}{|c|}{ Fluconazole } & \multicolumn{2}{c|}{ Itraconazole } & \multicolumn{2}{c|}{ Amphotericin B } \\
\hline & & $\mathrm{S}$ & $\mathrm{R}$ & $\mathrm{S}$ & $\mathrm{R}$ & $\mathrm{S}$ & $\mathrm{R}$ \\
\hline C.parapsilosis & 7 & $5(80 \%)$ & $2(20 \%)$ & $6(87 \%)$ & $1(13 \%)$ & $7(100 \%)$ & $0 \%$ \\
\hline C.krusei & 4 & $3(88 \%)$ & $1(12 \%)$ & $2(80 \%)$ & $2(20 \%)$ & $3(88 \%)$ & $1(12 \%)$ \\
\hline C.glabrata & 3 & $2(88 \%)$ & $1(12 \%)$ & $2(88 \%)$ & $1(12 \%)$ & $3(100 \%)$ & $0 \%$ \\
\hline C.kefyr & 2 & $1(50 \%)$ & $1(50 \%)$ & $1(50 \%)$ & $1(50 \%)$ & $2(100 \%)$ & $0 \%$ \\
\hline C.tropicalis & 1 & $1(100 \%)$ & $0 \%$ & $1(100 \%)$ & $0 \%$ & $1(100 \%)$ & $0 \%$ \\
\hline
\end{tabular}

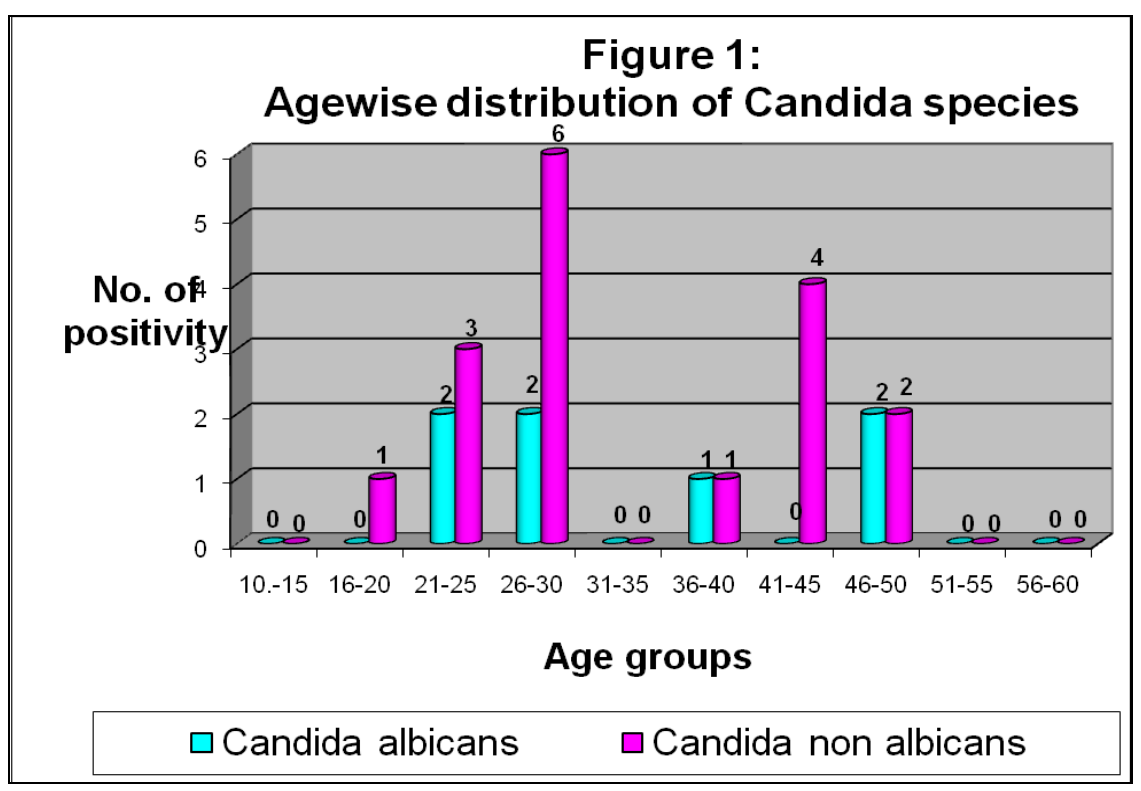

Figure 2:

Distribution of Candida species based on germ tube test

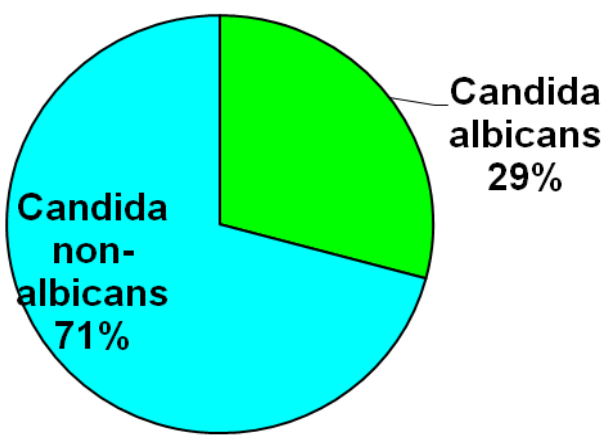




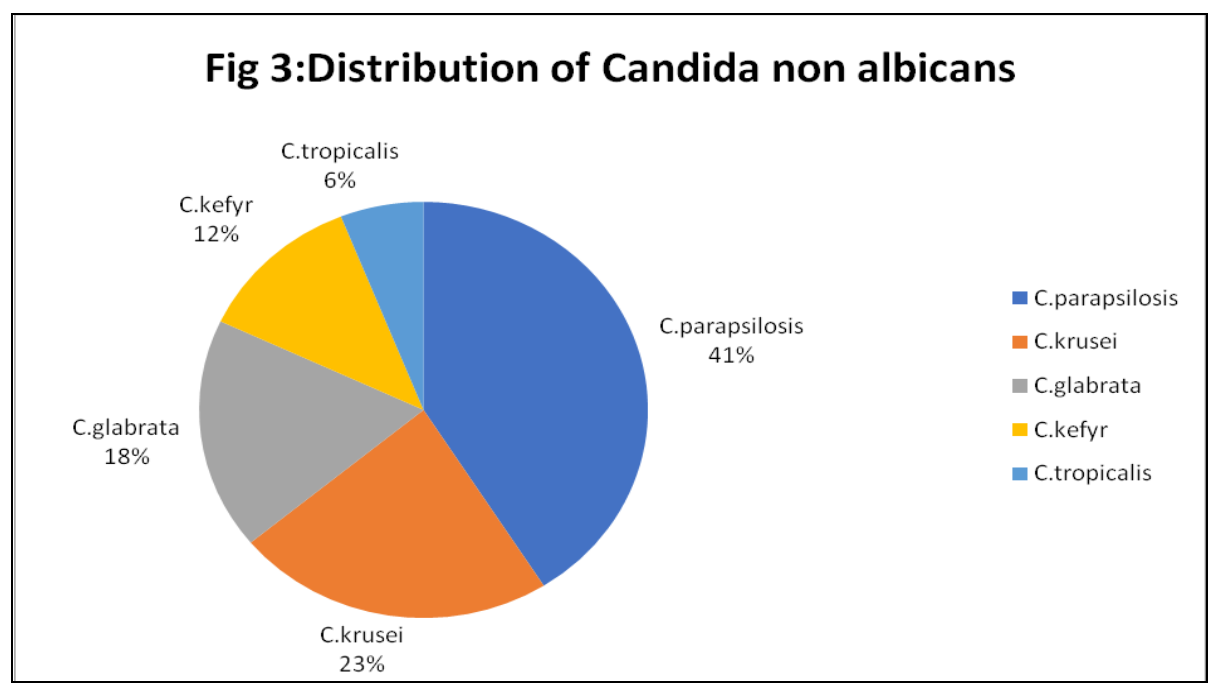

\section{Figure 4: PERCENTAGE OF DIABETIC $f$ NONDIABETIC PATIENTS}

$\begin{array}{cc}\text { 3rd Qtr } & \text { DIABETIC } \\ 0 \% & 1.5 \%\end{array}$

rigme 5:

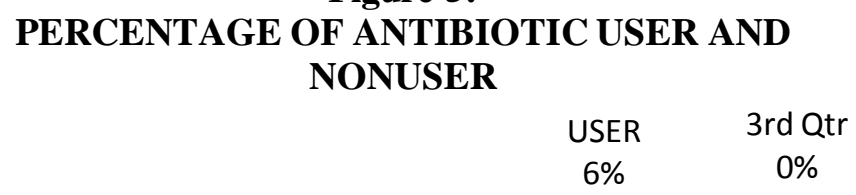

NONUSER 
Vaginal candidiasis is a common problem throughout the world and is a frequent disorder in Obstetrics and Gynaecology. Yeast may be present in the vagina despite the absence of clinical symptoms in up to $30 \%$ of patients. It has been estimated that about $3 / 4$ of all women suffer from at least one attack of this disease (Daniela et al., 1997).

In the present study, among the 68 samples collected $35 \% \quad(n=24)$ were found to grow Candida. In a study done by Jindal et al., $2006,23.4 \%$ ( $n=82$ of 350 women) was found to be positive for Candida species.

In the study, Candida albicans was isolated at the rate of $29 \%(n=7)$ and Candida non albicans $71 \%(\mathrm{n}=17)$. Daniela Arzeni et al., 1997 reported that the Candida non albicans were more prevalent than the Candida albicans in the symptomatic women with Jindal et al., 2006 reported 74\% Candida albicans and only $26 \%$ to be Candida non albicans. Likewise AlirezaKhosravi et al., 2010 and Richter et al., 2005 reported 65\% and $71 \%$ of Candida albicans in high vaginal swabs.

In the present study VVC was observed more in patients belonging to the age group 21 to 30 years. Similarly Alireza Khosravi et al., 2010 has also reported maximum prevalence in patients from 20 to 40 years of age. Sandra Richter et al., 2005 reported maximum prevalence of VVC in patients from the age group of 50 to 59 years.

In the present study, VVC was observed to be more in non pregnant women $63 \% \quad(n=43)$ than in the pregnant women $37 \%(n=25)$. Likewise David Trofa et al., 2008 also observed higher incidences of VVC among non pregnant women. Whereas Jindal et al., 2006 observed that the prevalence of VVC was more in pregnant women than in non pregnant women.
In the present study regarding the distribution of Candida non albicans species Candida parapsilosis $41 \%(n=7)$ was found to be the most prevalent species followed by Candida krusei $23 \%(\mathrm{n}=4)$, Candida glabrata $18 \%(\mathrm{n}=3)$, Candida kefyr $12 \%(\mathrm{n}=2)$ and Candida tropicalis 6\%(n=1).David Trofa et al., 2008 observed that among the Candida non albicans species Candida parapsilosis though rarely isolated in earlier days have found to be seen in increasing numbers now a days. Alireza Khosravi et al., 2010 observed that among the Candida nonalbicans species Candida tropicalis was found to be more prevalent followed by Candidaglabrata, Candida guilliermondii, Candida parapsilosis and Candida krusei.

Jindal et al., 2006 observed that out of the Candida non albicans Candida glabrata was found to be more prevalent followed by Candida tropicalis, Candida krusei, Candida parapsilosis and Candida guilliermondii. Most non-albicans Candida species have higher azole MICs and infections they cause are often difficult to treat. In present study also higher resistance was observed. One of the possible explanations for more frequent isolation of non-albicans species may be the increased use of topical azole agents. The extended prophylactic use of fluconazole in suspected cases would be a pro-bable cause of high resistance pattern to fluconazole in our institute. Another established fact is that antifungal drug response in vitro may be dose dependent which is expressed as susceptible dose dependent (SDD), that is, although susceptible in vitro but resistance failure may be seen in vivo at the usual dose. In such situations, increase in dose of drug above the usual dose often results in clinical cure. But Indian studies shown a very high resistance to fluconazole for all candidal isolates although the amphotericin B susceptibility is high, Adhikary (2011). 
In conclusion, vaginal Candidiasis affects about 50 to $72 \%$ of women, 40 to $50 \%$ having recurrent episodes. The majority of episodes of VVC are uncomplicated. Many women in whom recurrent yeast infections are diagnosed have been misdiagnosed. Recurrent episodes require clinical examination, culture of swabs, and consideration of underlying disease. Azoles can be used for empirical therapy of uncomplicated candidiasis as most of the isolates were found susceptible. However, culture should be done to detect non-albicans species and antifungal susceptibility testing is essential in recurrent cases of candidiasis.

\section{References}

Adhikary, R., Joshi, S. 2011. Species distribution and anti-fungal susceptibility of Candidaemia at a multi super-specialty center in Southern India. Indian J. Med. Microbiol., 29(3): 309311.

AlirezaKhosravi, Hojjatollah Shokri, R. Savadi, et al. 2010. A comparison study between the direct agglutination test \& conventional methods in the diagnosis of vulvovaginal candidiasis. DOI 10.1007/s00580-010-1050-1.

Ana, P., Silva, Isabel, M., Miranda, Carmen Lisboa, et al. 2009. Prevalence, distribution and antifungal susceptibility profiles of Candida parapsilosis, C.orthopsilosis and C. metapsilosis in a tertiary care hospital. J. Clin. Microbiol., p. 2392-2397.

Ariane Bruder-Nascimento, Carlos Henrique Camargo, Maria Fatima Sugizaki, et al. 2010. Species distribution and susceptibility profile of Candida species in a Brazilian public tertiary hospital. Bruder- Nascimento et al., BMC Res. Notes, 3: 1.

Daniela Arzeni, Maurizio Del Poeta, Oriana Simonetti, et al., Prevalence and antifungal susceptibility of vaginal yeast in outpatients attending a gynaecological center in Ancona, Italy. European J. Epidemiol., 13: 447-450.

David Trofa, Attila Gacser and Joshua D. Nosanchuk. 2008. Candida parapsilosis an emerging fungal pathogen. Clin. Microbiol. Rev., p: 606-625.

Dominique Sangland and Jacques Bille. 2002. Current understanding of the modes of action of and resistance mechanisms to conventional and emerging antifungal agents for the treatment of Candida infections.

Ella, M., de Leon, Scott, J., Jacober, Jack, D., Sobel and Betsy Foxman. 2002. Prevalence and risk factors for vaginal Candida colonization in women with type 1 and type 2 Diabetes. $B M C$ Infectious Dis., 2: 1.

Emma Shifrin, Debra Matityahu, Joseph Feldman and Howard Minkoff. 2000. Determinants of incident Vulvovaginal Candidiasis in Human Immunodeficiency Virus - positive women. Infectious Dis. Obstetrcs and Gynaecol., 8: 176-180.

Erna, M., Kojic and Rabih, O. 2004. Candida infections of medical devices. Clinical Microbiol. Rev., p. 255-267.

Fran Fisher. Fundamentals of Diagnostic Mycol.

Gary, P., Moran, Derek, J., Sullivan and David, C., Coleman. 2002. Emergence of Non Candida albicans Candida species as pathogens.

Jagdish Chander. Textbook of Medical Mycology, $3^{\text {rd }}$ edition.

Jindal, N., P. Gill, A. Aggarwal. An epidemiological study of Vulvovaginal Candidiasis in women of childbearing age. Indian J. Med. Microbiol., vol.25, No.2.

Malcolm, D., Richardson and Petteri Carlson. Culture and non culture based 2002. diagnostics for Candida species. 
Marie, V., Pirotta and Suzanne, M., Garland. 2006. Genital Candida species detected in the samples from women in Melbourne, Australia, before and after treatment with antibiotics. J. Clin. Microbiol., p. 3213-3217.

Mette, D., Jacobsen, Amanda, D., Duncan, Judith Bain, et al., Mixed Candida strain populations in colonized and infected mucosal tissues. DOI: $10.1111 / \mathrm{j} .1567-$ 1364.2008.00438.x.

Nabhan, A. 2006. Vulvovaginal Candidiasis. ASJOG, Vol3.

Paul Nyirjesy. 2001. Chronic Vulvovaginal Candidiasis. Jefferson Medical College, Pennsylvania, 63(4): 697-703.

Pfaller, M.A. and D.J. Diekema. 2007. Epidemiology of Invasive Candidiasis: a persistent public health problem. Clin. Microbiol. Rev., p.133-163.

Pfaller, M.A., D.J. Diekema and D.J. Sheehan. 2006. Interpretive breakpoints for fluconazole and Candida revisited: a blueprint for the future of Antifungal Susceptibility testing. Clin. Microbiol. Rev., p.435-447.

Ribeiro, M.A., R. Dietze, C.R. Paula et al. 2000. Susceptibility profiles of vaginal yeast isolates from Brazil. Mycopathologia, 151: 5-10.

Richard, A., Calderone. 2002. Taxonomy and Biology of Candida.

Stacy Tessler Lindau, Karl Mendoza, Hanna Surawska et al. 2008. Vaginal Candidiasis. NSHAP Biomeasures Technical Report.

Walter Magliani, Stefania Conti, Antonella Salati, et al. 2002. New strategies for treatment of Candida Vaginal Infections. Rev. Iberoam. Micol., 19: $144-148$.

White, D.J., A. Vanthuyne. 2006. Vulvovaginal Candidiasis. Sex Transm. Infect., 82(Suppl IV): iv 28-iv 30. DOI: 10.1136/sti.2006. 023168 .

\section{How to cite this article:}

Sangamithra, V., Susan, Radha Madhavan and Gomathi. 2017. Prevalence of Non albicans Candida Vaginal Infection among Women Attending the Obstetrics and Gynaecology Department of a Tertiary care Hospital. Int.J.Curr.Microbiol.App.Sci. 6(5): 1296-1305. doi: https://doi.org/10.20546/ijcmas.2017.605.140 